\title{
Magnetic and Dielectric Properties of BST/BF
}

\section{Multiferroic Ceramic Materials}

\author{
Yabo Zhang ${ }^{1}$, Pingsun Qiu ${ }^{2}$, Yubai Pan ${ }^{1}$, Jinrong Lin $^{1}$, Feifei Wang ${ }^{1}$, Yanxue Tang ${ }^{1}$, Xiyun $\mathrm{He}^{2}$ and Dazhi Sun ${ }^{1 *}$ \\ 1. Department of Chemistry, Shanghai Normal University, 100 Guilin Road, Shanghai 200234, China \\ 2. Shanghai Institute of Ceramics, Chinese Academy of Sciences, 1295 DingxiRoad,Shanghai 200050, China
}

\begin{abstract}
The lead-free multiferroic ceramic of $(1-\mathrm{x}) \mathrm{Ba}_{0.70} \mathrm{Sr}_{0.30} \mathrm{TiO}_{3}(\mathrm{BST})+\mathrm{xBiFeO}_{3}(\mathrm{BFO})$ was synthesized by a conventional solid-state reaction method. The dielectric, ferroelectric and magnetic properties was studied. The X-ray diffractomentry (XRD) revealed that the ceramic formed a single perovskite structure. The results suggested that the remanent magnetization (Mr) reached to maximum when the BFO content is up to 0.05 . The powder of the samples $\mathrm{Mr}=70 \mathrm{memu} / \mathrm{g}$ when $\mathrm{x}=0.05$. The change of the electrical resistance with magnetic field of the (1-x) $\mathrm{Ba}_{0.70} \mathrm{Sr}_{0.30} \mathrm{TiO}_{3}+\mathrm{xBiFeO}_{3}$ samples reached $25.6 \%$ when $\mathrm{x}=0.03$. The relative dielectric constant was reduced under magnetic field compared with no magnetic field and the change is $13.1 \%$ as $\mathrm{x}=0.01$. It is indicated that the lead-free multiferroic ceramic of $(1-\mathrm{x}) \mathrm{Ba}_{0.70} \mathrm{Sr}_{0.30} \mathrm{TiO}_{3}+\mathrm{xBiFeO}_{3}$ exhibited both ferroelectric and magnetic at room temperature. The ferroelectric and magnetic properties are derived from the displacement of magnetic atom iron and the change of the atomic spin state.
\end{abstract}

Key words: Multiferroic, dielectric, ferroelectric, BST.

\section{Introduction}

Multiferroic magnetoelectrics are materials that both ferromagnetic/antiferromagnetic and ferroelectric/antiferroelectric properties exist in the same phase. These materials have a magnetoelectric effect that electric polarization can be induced in the material by an applied magnetic field and magnetization is induced by an applied electric effect, that is magnetoelectric coupling in the multiferroic materials [1]. This is difficult to be synthesized because ferroelectricity and ferromagnetism make a mutually exclusive group [2]. But the magnetoelectric coupling of magnetic and electrical properties is a more general and widespread phenomenon. As this new century unfolds, the study of materials possessing coupled magnetic and electrical order parameters has been revitalized [3, 4].

Barium strontium titanate $\mathrm{Ba}_{1-\mathrm{x}} \mathrm{Sr}_{\mathrm{x}} \mathrm{TiO}_{3}$ is a solid solution composed of barium strontium and strontium

*Corresponding author: Dazhi Sun, professor, research field: functional materials. titanate. When barium atoms are replaced by strontium atoms at A site in perovskite barium titanate matrix, the phase transition temperature of paraelectric to ferroelectric decreases and the phase transition behavior changes from sharp to diffuse [5-7]. Among the perovskites mixed systems, $\mathrm{Ba}_{1-\mathrm{x}} \mathrm{Sr}_{\mathrm{x}} \mathrm{TiO}_{3}$ (BST) is an interesting series due to its unique ferroelectric properties which are suitable for various potential applications [8]. And that the BST system shows high permittivity which has a wide interest in the field of electrically controlled devices [9].

Bismuth ferrite $\left(\mathrm{BiFeO}_{3}\right)$ is one of the few known single-phase multiferroic materials which exhibits rhombohedrally distorted perovskite structure. The parameters of the hexagonal elementary cell are: $\mathrm{a}=$ $5.5876 \AA$ and $\mathrm{c}=13.867 \AA$ at room temperature. It has antiferromagnetic ordering with $\mathrm{T}_{\mathrm{N}} \sim 370{ }^{\circ} \mathrm{C}$ and ferroelectric properties with a high Curie temperature of about $\sim 830 \quad{ }^{\circ} \mathrm{C} \quad[10] . \quad \mathrm{BiFeO}_{3}$ has showed magnetoelectric coupling. But there are some obstacles need to be overcome before to be applied. Firstly, it is also difficult to control oxygen 
stoichiometry and the evaporation of bismuth oxide in the sample. Secondly, the $\mathrm{BiFeO}_{3}$ bulk material exists the large leakage current since the existence of the mixed valence $\mathrm{Fe}^{2+} / \mathrm{Fe}^{3+}$ ions and oxygen vacanvies $[11,12]$. Thirdly, the $\mathrm{BiFeO}_{3}$ shows very weak even no ferromagnetism from a near-linear magnetization-magnetic field $(\mathrm{M}-\mathrm{H})$ relation [13]. So it is proposed that the compound of a solid splution with BST compositions.

It is said that the structure of $\mathrm{Ba}_{0.7} \mathrm{Sr}_{0.3} \mathrm{TiO}_{3}$ is difficult to decide whether it is tetragonal or cubic because of the large uncertainties after final refinement [14], and we choose $\mathrm{Ba}_{0.7} \mathrm{Sr}_{0.3} \mathrm{TiO}_{3}$ and $\mathrm{BiFeO}_{3}$ as research object so that we could use the properties to study the magnetoelectric coupling.

In this work, it is studied that the magnetic and electrical properties of (1-x)BST $+\mathrm{xBiFeO}_{3}$ system and the effect of $\mathrm{BiFeO}_{3}$ doping on the formation of the $(1-\mathrm{x}) \mathrm{BST}+\mathrm{xBiFeO}_{3}$ solid solution. It was found that the (1-x)BST $+\mathrm{xBiFeO}_{3}$ series ceramic both have ferroelectric and ferromagnetic at room temperature. With the increase of BFO doping amount, ceramic remanent magnetization first increase then decrease. When $\mathrm{x}=0.05$, the remanent magnetization of the sample powder reached maximum that is $\mathrm{Mr}=70$ $\mathrm{memu} / \mathrm{g}$ at this time. When $\mathrm{x}=0.01$, the largest of the remanent polarization is $\operatorname{Pr}=11 \mu \mathrm{C} / \mathrm{cm}^{2}$. And after applied magnetic field, the relative dielectric constant of ceramic material decreases, exhibiting obvious electro-magnetic coupling behavior.

\section{Experimental Methods}

The (1-x) $\mathrm{Ba}_{0.70} \mathrm{Sr}_{0.30} \mathrm{TiO}_{3}(\mathrm{BST})+\mathrm{xBiFeO}_{3}$ ceramic system was prepared by a conventional solid-state reaction process. The addition of $\mathrm{BiFeO}_{3}$ is $\mathrm{x}=0.01$, 0.03, 0.05 and 0.07, respectively. Commercial $\mathrm{BaCO}_{3}$, $\mathrm{SrCO}_{3}, \mathrm{TiO}_{2}, \mathrm{Bi}_{2} \mathrm{O}_{3}$ and $\mathrm{Fe}_{2} \mathrm{O}_{3}$ powders all were of purities greater than $99.9 \%$. Raw powders according to the stoichiometric ratio were ball milled with ethylalcohol for $6 \mathrm{~h}$. After the mixture was dried, it was calcined at $1,150{ }^{\circ} \mathrm{C}$ for $2 \mathrm{~h}$ in air. Then after the second ball milled, the calcined powders were mixed with 6\% PVA binder and pressed into cylindrical pellets of $15 \mathrm{~mm}$ diameter. Samples were sintered at 5 ${ }^{\circ} \mathrm{C} / \mathrm{min}$ to $1260^{\circ} \mathrm{C}$, with a $2 \mathrm{~h}$ hold at $1,260^{\circ} \mathrm{C}$. Phase identification of these specimens was conducted by X-ray diffractomentry (XRD) (D8 ADANCED XRD, BRUKER/AXS Co., Germany) with $\mathrm{Cu}$ Ka radiation. Data was collected from $20^{\circ}$ to $80^{\circ}$ of $2 \theta$. A scanning electron microscope (SEM) (JEOL-JSM-6460, Japan) was used to detect the micromorphology. Silver paste was deposited on the surface of the pellet. The ferroelectric properties were examined by p-e loop (MTI 2000). The magnetic properties of the samples were measured by VSM (Lakeshore 7400). The permittivity was investigated at $1 \sim 300 \mathrm{KHz}$ by $\mathrm{TH} 2818$ Automatic Component Analyzer.

\section{Results and Discussion}

Fig. 1 shows the XRD spectra of (1-x)BST $+\mathrm{xBFO}$ $(\mathrm{x}=0.01,0.03,0.05$ and 0.07$)$ ceramics were measured with a $\mathrm{Cu}$ Ka source at room-temperature. The sintering temperature is $1,260{ }^{\circ} \mathrm{C}$. It is found that the samples have no other phase diffraction peak and exhibit single perovskite structure. BST and BFO formed a good solid solution and did not change the structure. $\mathrm{Bi}$ ions and $\mathrm{Fe}$ ions entered the lattice of $\mathrm{BST}$, instead of $\mathrm{Ba}$ and $\mathrm{Sr}$ ions at A site. Because the ionic radius of $\mathrm{Bi}$ and $\mathrm{Fe}$ is smaller than $\mathrm{Ba}$ and $\mathrm{Sr}$, the distance between the oxygen atoms is reduced, further the crystal lattice constant is reduced, and the $\mathrm{d}$-spacing $\mathrm{d}$ decreases. According to Bragg equation:

$$
2 d \sin \theta=n \lambda
$$

the diffraction Angle theta decreases with d decreases, and the intensity of the diffraction peakenhances. So the diffraction peak move slightly to the right.

Fig. 2 shows the magnetic hysteresis loops of $(1-\mathrm{x}) \mathrm{BST}+\mathrm{xBFO}(\mathrm{x}=0.01,0.03,0.05$ and 0.07$)$ powder at room temperature. The saturated magnetizing strength and the remanence firstly increased and then decreased with the increment of BFO. 


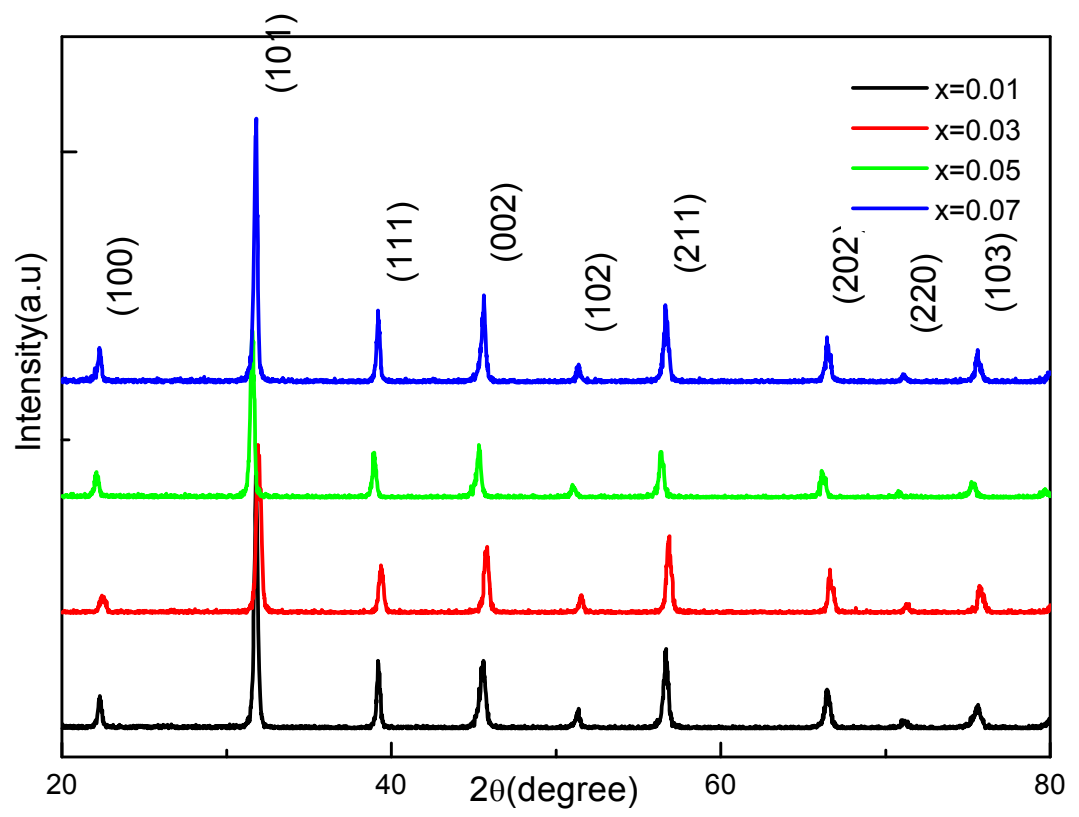

Fig. 1 XRD spectra of $(1-x) B S T+x B F O(x=0.01,0.03,0.05$ and 0.07$)$.

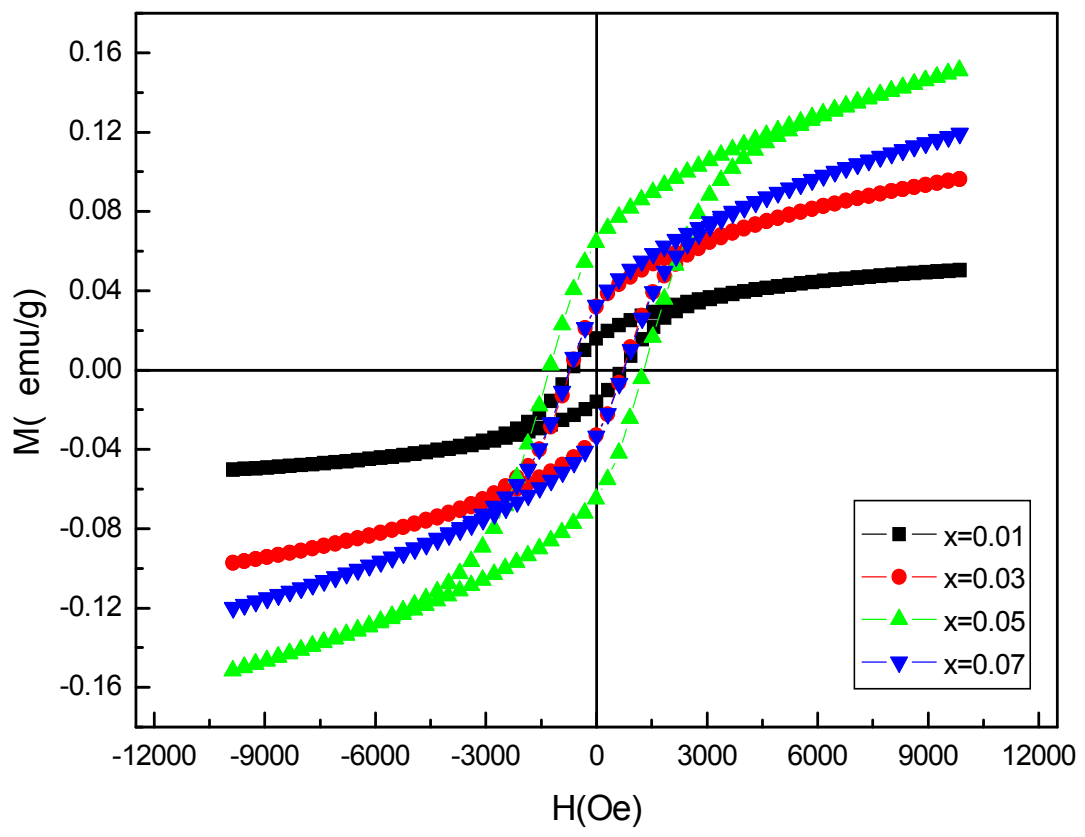

Fig. 2 Magnetic hysteresis loops of $(1-x) B S T+x B F O(x=0.01,0.03,0.05$ and 0.07$)$ powder.

The remanent magnetization $(\mathrm{Mr})$ reached maximum when the doping amount of BFO is up to 0.05. The Mr $=70 \mathrm{memu} / \mathrm{g}$ when $\mathrm{x}=0.05$. It is indicated that the powder hold good ferromagnetic at room temperature. $\mathrm{BiFeO}_{3}$ is perovskite materials with type $\mathrm{G}$ antiferromagnetic structure, and the relative displacement of $\mathrm{Bi}^{3+}$ causes uneven crystal structure, so that it arranged in spiral structure, and performance weak ferromagnetic on a macro. Barium strontium titanate is perovskite ferroelectric materials, and $\mathrm{Fe} \mathrm{Bi}$ ions can enter into oxygen octahedral structure of BST, destroyed the ferrite bismuth spiral magnetic structure.

Fig. 3 shows the electric hysteresis loops of $(1-\mathrm{x}) \mathrm{BST}+\mathrm{xBFO}(\mathrm{x}=0.01,0.03,0.05$ and 0.07$)$ ceramics. We can know the leakage current is too large from this image except $\mathrm{x}=0.03$. The remanent 
polarization reached $12 \mu \mathrm{C} / \mathrm{cm}^{2}$ as $\mathrm{x}=0.01$. As we can see from the Fig. 3, these samples exhibited large leakage current. It is easy that Bi elements volatiled under thigh temperature. In this case, it caused the crystal defect.

Fig. 4 shows the insulation resistance of $(1-\mathrm{x}) \mathrm{BST}+$
$\mathrm{xBFO}(\mathrm{x}=0.01,0.03,0.05$ and 0.07$)$ ceramics under the condition of magnetic field (the red line) and with no magnetic field (the black line). First the resistance decreased after add the magnetic field and then increased slightly. When $x=0.03$, the change of the resistance is $25.6 \%$. The magnetic field make the ceramic

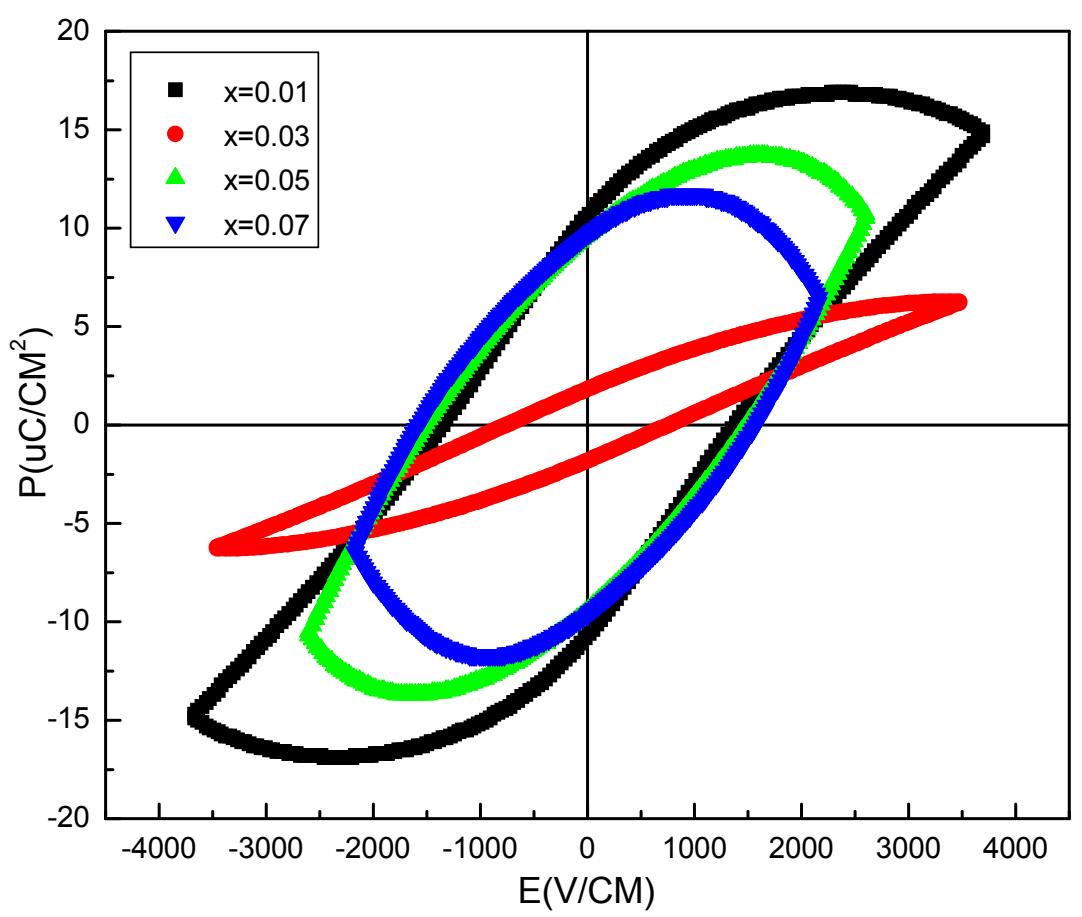

Fig. 3 Electric hysteresis loops of $(1-x) B S T+x B F O(x=0.01,0.03,0.05$ and 0.07$)$ ceramics.

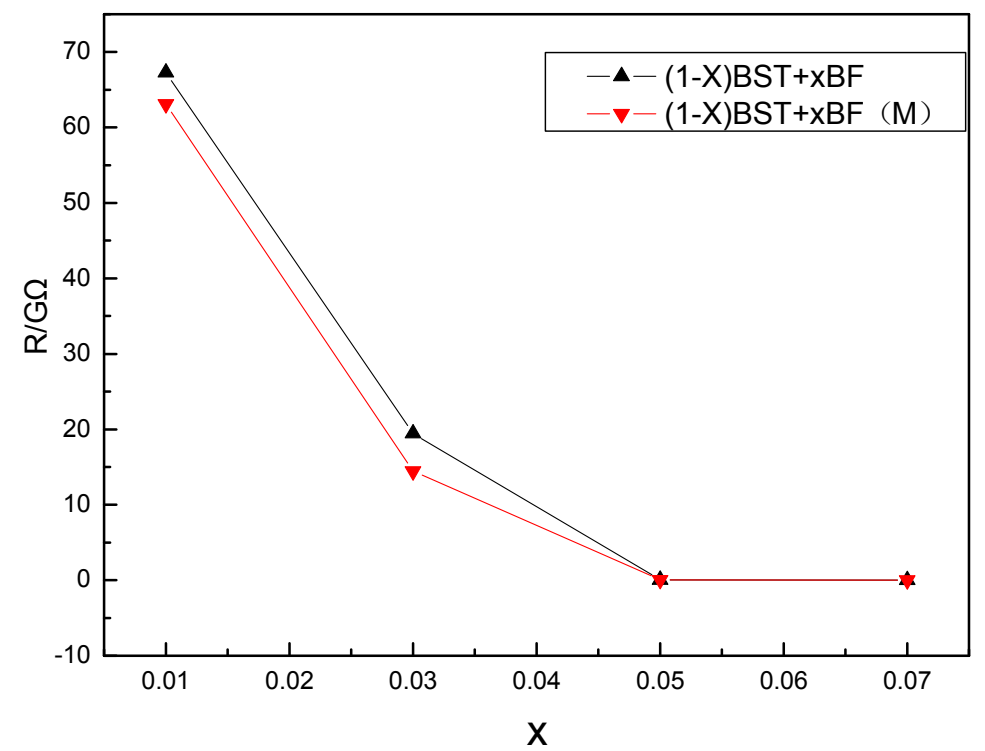

Fig. 4 Resistance of (1-x)BST + xBFO $(x=0.01,0.03,0.05$ and 0.07$)$. 
grain oriented lead to the resistance drop at the beginning. When $\mathrm{BiFeO}_{3}$ reaches a certain limit, the number of crystal orientation with different increase, the grain boundary changed a lot, the grain boundary resistance is bigger, the resistance of the charge movement increase.

Fig. 5 shows the contrast of the relative dielectric constant with frequency of (1-x)BST $+x B F O(x=$ $0.01,0.03,0.05$ and 0.07$)$ ceramics. Compared with no magnetic field, the relative dielectric constant was reduced under magnetic field. When $\mathrm{x}=0.01$, the change is biggest. At this point, the change is about $13.1 \%$. Under the condition of magnetic field, the magnetic field force acting on domain boundary make the mobility of domain boundary reduce and domain's
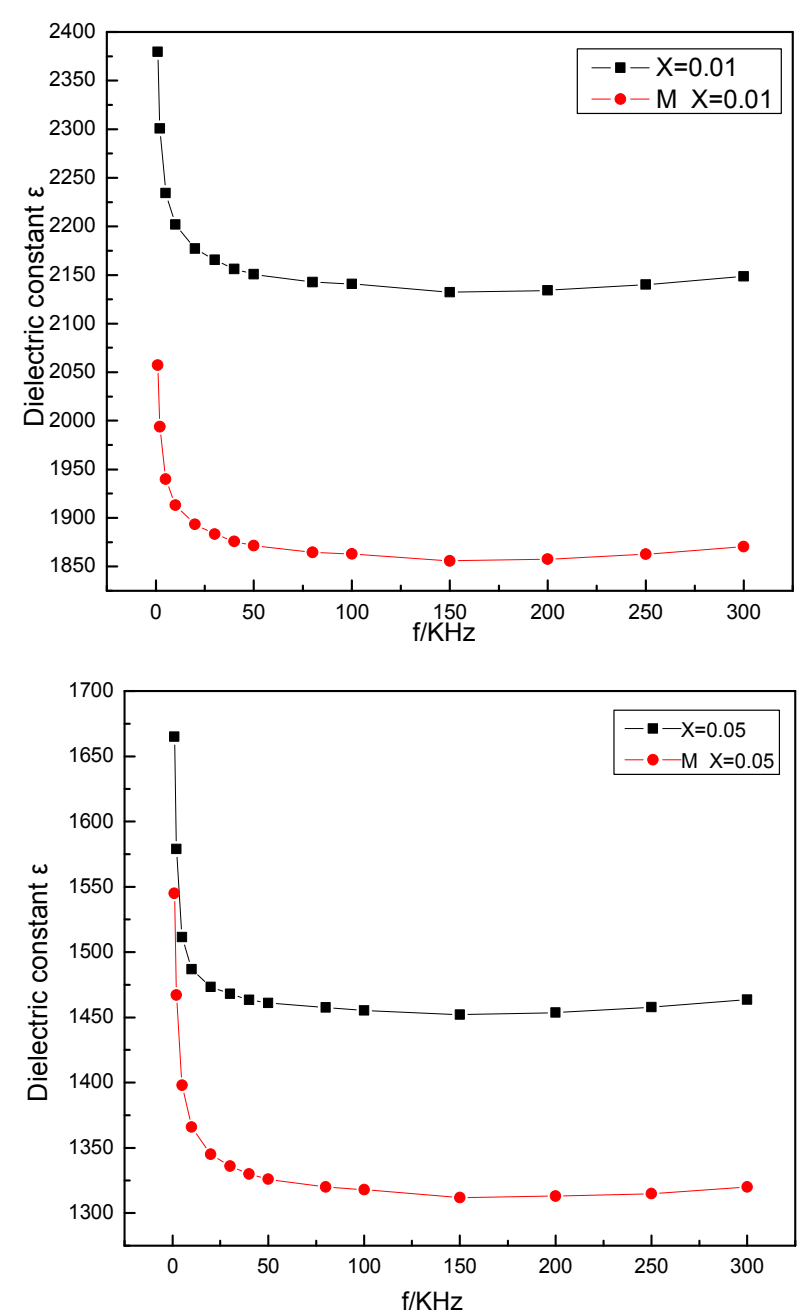

contribution on the dielectric constant decreases, and make the dielectric loss decrease.

Fig. 6 shows the contrast of the dielectric loss with frequency of (1-x)BST $+\mathrm{xBFO}(\mathrm{x}=0.01,0.03,0.05$ and 0.07$)$ ceramics. Compared with no magnetic field, the relative dielectric constant increased slightly under magnetic field. But it is reduced when $\mathrm{x}=0.03$. The change of the dielectric loss reached $28.2 \%$ at 20 KHz. The reasons for the results may be the structure of the material that in the vicinity of the phase boundary changed by a strong magnetic field, resulting in increase of the dielectric response. Moreover, the clamping action of magnetic make the contribution of domain boundaries of the domain of dielectric loss reduce, significantly reducing the dielectric loss.
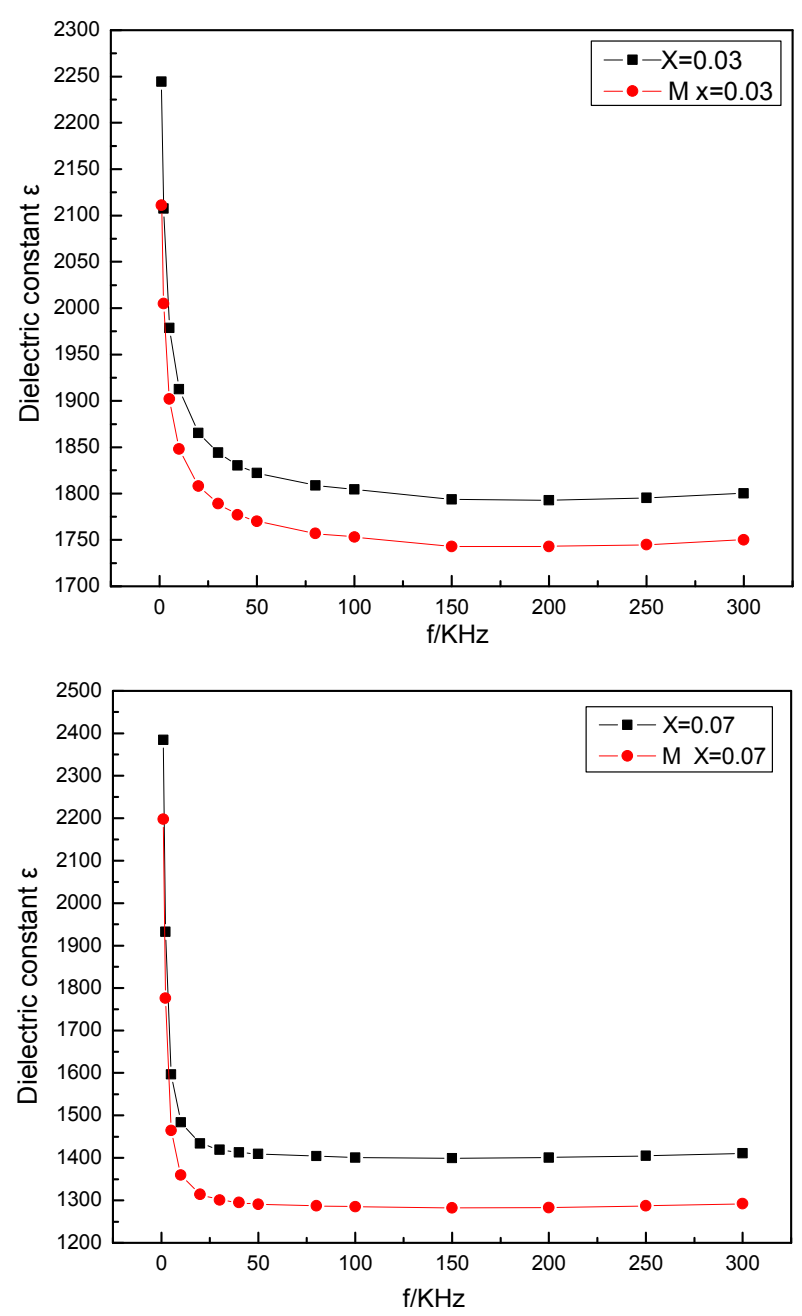

Fig. 5 The relative dielectric constant with frequency of $(1-x) B S T+x B F O(x=0.01,0.03,0.05,0.07)$. 

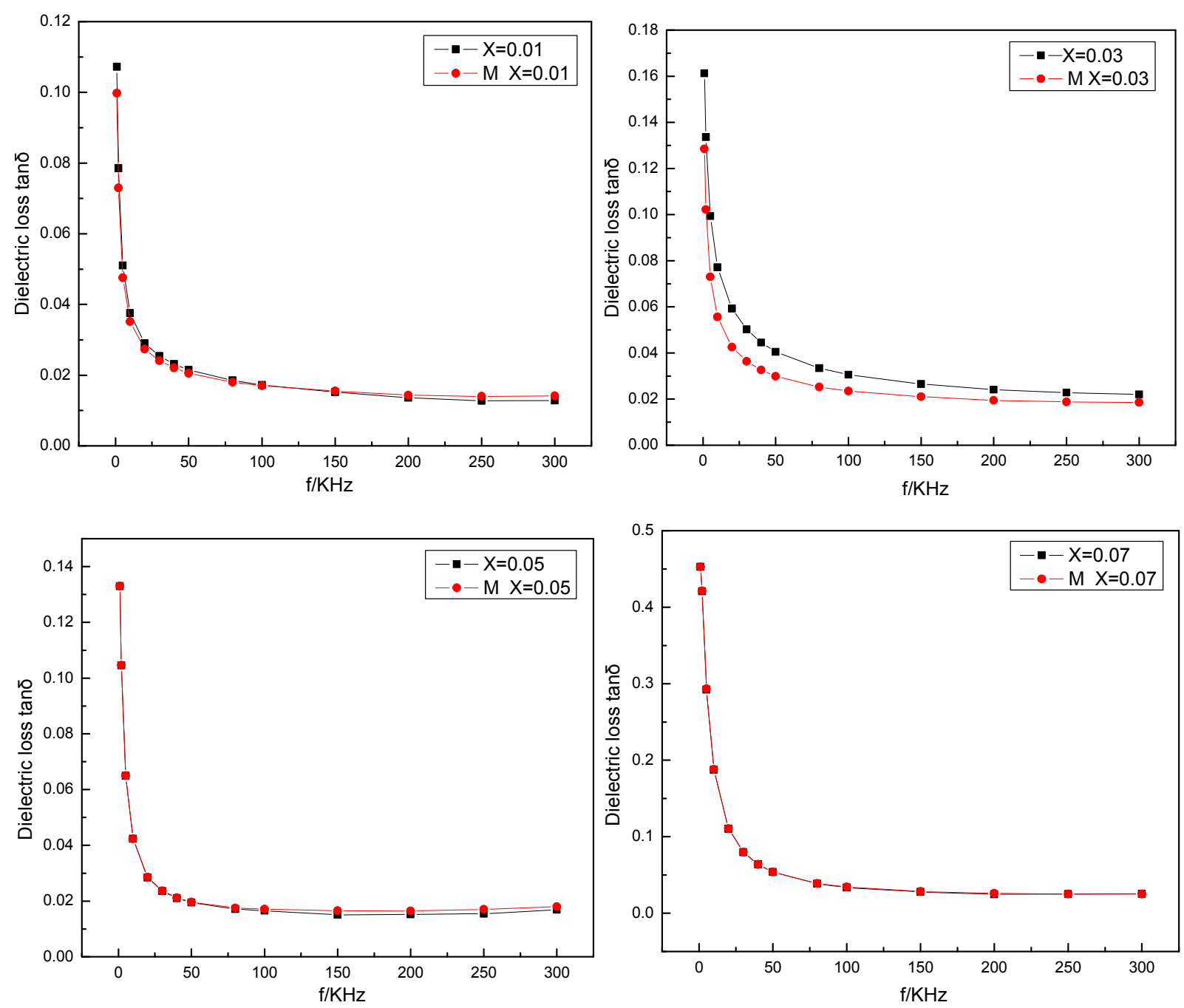

Fig. 6 The dielectric loss with frequency of $(1-x) B S T+x B F O(x=0.01,0.03,0.05$ and 0.07$)$ ceramics.

\section{Conclusions}

In general, the $(1-\mathrm{x}) \mathrm{BST}+\mathrm{xBiFeO}_{3}$ ceramics exhibit single perovskites structure. The samples have both ferroelectricity and ferromagnetism at room temperature. The dielectric constant of ferroelectric materials change evidently at the phase temperature and arise the peak even reach the highest value at curie temperature $(\mathrm{Tc})$. In this case, the structure relaxation leads to lower activation energy of ions. So the new domains can formed spontaneously as the ions have larger mobility. The domains turn easily under the added electric field. When it is magnetized by external magnetic field, makes the lattice deformation occur in each domain. The content of
$\mathrm{BiFeO}_{3}$ from $1 \%$ to $3 \%$ can result in obvious magnetoelectric coupling effect, but when excess $\mathrm{BiFeO}_{3}$ could easily produce the defects of iron clusters lead to make smaller dielectric response, and related research is underway. The results suggest that the lead-free material is important to new magnetoelectric coupling and multiferroic materials and it could be widely used in many fields in the future.

\section{Acknowledgements}

The authors would like to thank the National Key R \& D Program of China (2016YFB0701004), the Science and Technology Commission of Shanghai Municipality (14ZR1430400, 16070502700), and the 
Key Laboratory of Inorganic Functional Materials and Devices of Chinese Academy of Sciences (KLIFMD-2015-07) for supporting the research.

\section{References}

[1] Zhou, Z.-Y., Yang, Q., Liu, M., Zhang, Z.-G., Zhang, X.-Y. and Sun, D.-Z. et al. 2015. "Antiferroelectric Materials, Applications and Recent Progress on Multiferroic Heterostructures.” SPIN 5 (1): 1530001.

[2] Hill, N. A. 2000. "Why are there so Few Magnetic Ferroelectrics?" Journal of Physical Chemistry B 104: 6694-709.

[3] Eerenstein, W., Mathur, N. D. and Scott, J. F. 2006. "Multiferroic and Magnetoelectric Materials." Nature 442: 759-65.

[4] Zhou, Z., Zhang, X. Y., Xie, T. F., Nan, T. X., Gao, Y. and Yang, X. et al. 2014. "Strong Non-Volatile Control of Magnetism in Magnetic/Antiferroelectric Magnetoelectric Heterostructures.” Applied Physics Letters. 104: 012905.

[5] Kukretic, A., Kumar, A. and Naithani, U. C. 2009. "Electric Field Dependence of Specific Heat in $\mathrm{Ba}_{\mathrm{x}} \mathrm{Sr}_{1-\mathrm{x}} \mathrm{TiO}_{3}$ Ferroelectric Perovskites." Indian Journal of Pure \& Applied Physics 47 (01): 43-8.

[6] Sengupta, L. C., Ngo, E., Synowezynski, J. and Sengupta, S. 1996. "Optical and Electrical Studies of Novel Ferroelectric Composites for Use in Phased Array Antennas." In Proceedings of the $10^{\text {th }}$ IEEE International Symposium on Applied Ferroelectrics, IEEE, New York, 845 .
[7] Wei, X. Y. and Yao, X. 2003. "Nonlinear Dielectric Properties of Barum Strontium Titanate Ceramics." Materials Science and Engineering B 99: 74-8.

[8] Zhang, J. J. and Zhai, J. W. 2009. "Microwave and Infrared Dielectric Response of Tunable $\mathrm{Ba}_{1-\mathrm{x}} \mathrm{Sr}_{\mathrm{x}} \mathrm{TiO}_{3}$ Ceramics." Science Direct Acta Material 57: 4491-9.

[9] Alexandru, H. V., Berbecaru, C., Stanculescu, F., Ioachim, A., Banciu, M. G. and Toacsen, M. I. et al. 2005. "Ferroelectric Solid Solutions $(\mathrm{Ba}, \mathrm{Sr}) \mathrm{TiO}_{3}$ for Microwave Applications." Materials Science and Engineering B 118: 92-6.

[10] Kubel, F. and Schmid, H. 1990. "Structure of a Ferroelectric and Ferroelastic Monodomain Crystal of the Perovskite $\mathrm{BiFeO}_{3}$." Acta Crystallographica Section B: Structural Science 46: 698-702.

[11] Jo, S. H., Lee, S. G. and Lee, Y. H. 2007. "Ferroelectric Properties of PZT/BFO Multilayer Thin Films Prepared Using the Sol-Gel Method." Nanoscal Research Letters 7: $1-5$.

[12] Kumar, M. Mashesh, Sriniras, A. and Suryanarayana, S. V. 2000. "Structure Properties Relations in $\mathrm{BiFeO}_{3} / \mathrm{BaTiO}_{3}$ Solid Solutions." Journal of Applied Physics 87: 855-62.

[13] Kim, J. S., Cheon, C. L., Chai, Y. N. and Jang, P. W. 2003. "Ferroelectric and Ferromagnetic Properties of $\mathrm{BiFeO}_{3}-\mathrm{PrFeO}_{3}-\mathrm{PbTiO}_{3}$ Solid Solutions." Journal of Applied Physics 93: 9263-70.

[14] Kim, S. W., Choi, H. I., Lee, M. H., Park, J. S., Kim, D. J. and Do, D. et al. 2013. "Electrical Properties and Phase of $\mathrm{BaTiO}_{3}-\mathrm{SrTiO}_{3}$ Solid Solution." Ceramic International 39: S487-90. 